W stużbie tradycji i odnowy liturgicznej. 50 lat Instytutu Liturgicznego w Krakowie (1968-2018), red. P. Nowakowski, J. Mieczkowski, Kraków 2019, s. 143-150.

ISBN 978-83-7438-849-8 (wersja drukowana), ISBN 978-83-7438-850-4 (wersja online)

DOI:http://dx.doi.org/10.15633/9788374388504.12

Alberto Vernaschi CM

Wincentyóskie Centrum Liturgiczne w Rzymie, WŁochy

\title{
Attività del Centro Liturgico Vincenziano di Roma
}

Ringrazio gli organizzatori del Convegno per avermi invitato a parlare del Centro Liturgico Vincenziano di Roma e rivolgo un cordiale saluto a tutti i partecipanti.

Tracciando le tappe essenziali della storia dell'Accademia Liturgica, P. Guerra è arrivato fino al decennio 1970-1980. La storia del Centro Liturgico Vincenziano (CLV) inizia con la sua ufficiale costituzione avvenuta il 31 gennaio 1978. Si tratta di un'Associazione, composta finora da missionari vincenziani, riconosciuta a livello civile italiano, con finalità proprie, che vengono così descritte nello Statuto:

Promuovere gli studi liturgici e il movimento liturgico attraverso la pubblicazione, la stampa e la diffusione di riviste e libri a carattere religioso, scientifico, pastorale e divulgativo; promuovere gli studi vincenziani e il movimento vincenziano attraverso la pubblicazione, la stampa e la diffusione di riviste e libri di ricerca, spiritualità e azione vincenziana; organizzare e collaborare a corsi di studio e di spiritualità liturgica e vincenziana.

Ma che cosa fa concretamente il Centro Liturgico Vincenziano al servizio della Liturgia?

1. Innanzitutto il CLV porta avanti la pubblicazione e la diffusione della rivista "Ephemerides Liturgicae", della quale, nel 1989, è uscito l'utile volume dell'Indice Generale dal 1887 - anno della sua fondazione - al 1986. 
La rivista, con le sue specifiche caratteristiche, continua il suo cammino, attualmente sotto la direzione del P. Giuseppe Guerra, con la preziosa collaborazione - da anni - di P. Anthony Ward come Segretario di redazione e con un nuovo Consiglio di Redazione. Questo si riunisce varie volte durante l'anno per programmare i fascicoli della rivista e valutare i vari contributi, secondo la metodologia del peer rewiew system. Del coetus dei consultori fa parte anche P. Przemysław Nowakowski, C.M.

2. Tra le opere di carattere generale pubblicate dal CLV meritano un particolare riguardo i quattro volumi dell' Enchiridion Documentorum Instaurationis Liturgicae (fino al 2003), preceduti dal volume Documenta ad Instaurationem liturgicam spectantia (1903-1963).

3. Alla rivista "Ephemerides Liturgicae" è legata la Collana BEL (Bibliotheca Ephemerides Liturgicae). Di essa fanno parte ben 188 volumi, comprendenti studi vari, comunque sempre di indole scientifica, riguardanti la liturgia o ambiti ad essa strettamente legati. Possiamo trovare nella Collana ricerche di studiosi di Liturgia di varie parti del mondo, gli Atti di alcuni anni delle Semaines d'études liturgiques delle Conférences Saint-Serge di Parigi (passati, purtroppo, da qualche anno ad altra casa editrice), gli Atti delle Settimane di Studio dell' APL, ossia dell' Associazione dei Professori di Liturgia (Studi di Liturgia), alcune tesi dottorali di particolare interesse del PIL, il Pontificio Istituto Liturgico di Sant'Anselmo di Roma (Liturgica Opera Prima). Logicamente, tutti questi testi entrano a far parte della Collana BEL soltanto se garantiti, quanto a scientificità e opportunità, dai responsabili della Collana stessa, o dall'APL, o dal PIL.

Ricordando qualche autore in particolare non vorrei far torto ad altri, ma non posso non accennare al testo fondamentale di Annibale Bugnini, La riforma liturgica (1948-1975), pp. 1002. Di mons. Bugnini, a 30 anni dalla sua morte, sono state pubblicate nel 2012 le Memorie autobiografiche dal titolo "Liturgiae cultor et amator" servi la Chiesa. Testi quanto mai documentati e importanti per la "vera storia" di mons. Bugnini e della riforma liturgica del Concilio Vaticano II.

Di grande rilievo i vari contributi di Carlo Braga, stretto collaboratore di mons. Bugnini ed estensore di non pochi testi liturgici durante il Concilio e nella fase applicativa del post-concilio. Tra le sue pubblicazioni che figurano nella Collana BEL ricordiamo: La riforma liturgica di Pio XII, Documenti, pp. 800 e La liturgia delle ore al Vaticano II, pp. 224. 
Ricordo ancora Vincenzo Raffa, Liturgia eucaristica. Mistagogia della Messa: dalla storia e dalla teologia alla pastorale pratica, pp. 1308; Enrico Cattaneo, Il culto cristiano in occidente, note storiche, pp. 662; Burkhard Neunheuser, Storia della liturgia attraverso le epoche culturali, pp. 220; Aimé Georges Martimort, Les diaconesses. Essai historique, pp. 278; Salvatore Marsili, o.s.b., I segni del mistero di Cristo. Teologia liturgica dei sacramenti, pp. 526; Vincenzo Raffa, Liturgia eucaristica. Mistagogia della Messa: dalla storia e dalla teologia alla pastorale pratica, pp. 1308; Sandrino Bocchin, La verginità professata, celebrata, confessata, pp. 1076; Paolo Tomatis, Accende lumen sensibus: La liturgia e i sensi del corpo, pp. 586; Enrico Mazza, L'anafora eucaristica. Studi sulle origini, pp. 398; Pietro Angelo Muroni, L'ordine dei sacramenti dell'iniziazione cristiana, pp. 510; Angelo Lameri, La "Pontificia Commissio de sacra liturgia praeparatoria Concilii Vaticani II". Documenti, Testi, Verbali, pp. 882; Pierangelo Chiaramello, Il rinnovamento liturgico cuore del rinnovamento della Chiesa nei Discorsi di Paolo VI (1963-1978), pp. 400; i tre volumi di AA. VV. dal titolo Celebrare il Mistero di Cristo, che costituiscono ottimi manuali per l'insegnamento della Liturgia. Tra i testi pubblicati più di recente, vale la pena ricordare Markus Tymister, La concelebrazione eucaristica. Storia, Questioni teologiche, Rito, pp. 330, di cui uscirà a breve la seconda edizione; Nikolaj Aracki Rosenfeld, Celebrare l'alleanza. La tipologia dalla Bibbia alla Liturgia, pp. 536; Giuseppe Midili, La riforma liturgica nella Diocesi di Roma. Studio in prospettiva storica e pastorale (1956-1975), pp. 426; AA.VV., Donne e uomini: il servizio nella liturgia, pp. 276; Daniel-Alberto Escobar Portillo, Te Deum laudamus. La formulación y la celebración de la gloria de Dios a través de una forma hímnica, pp. 308; Sante Ippoliti, La soteriologia liturgica sullo sfondo della teologia drammatica di Hans Urs von Balthasar, pp. 326. Ma... dovrei segnalarli tutti!

Alla Collana BEL sono inoltre legati i Subsidia "Instrumenta Liturgica Quarreriensia" Supplementa (finora cinque): si tratta di riedizioni critiche di testi liturgici di particolare interesse.

Vi è inoltre la Collana Cantate et Psallite, per la Formazione al canto liturgico, di cui sono usciti 4 volumi.

Una serie minore, ma molto interessante e utile, è costituita dai 26 testi dei Sussidi Liturgico-Pastorali, alcuni particolarmente pregevoli.

Il CLV si occupa pure della pubblicazione degli Atti delle Settimane Liturgiche nazionali italiane organizzate dal CAL (= Centro di Azione Liturgica) dal 1989 ad oggi. 
Inoltre, dal 2016, è stata affidata al CLV la pubblicazione della Collana Caro Salutis Cardo dell'Istituto di Liturgia Pastorale di Santa Giustina di Padova.

Non manca poi la pubblicazione di opere singole, al di fuori dei circuiti sopra ricordati.

4. Al settore liturgico si affianca quello vincenziano. In esso confluiscono una serie di pubblicazioni che riguardano San Vincenzo de' Paoli, Santa Luisa de Marillac, Santi e Beati della Famiglia Vincenziana, libri vari di formazione vincenziana.

Di particolare rilievo la pubblicazione, in atto, delle Opere di San Vincenzo de' Paoli in una nuova edizione critica: usciti finora 5 volumi della Corrispondenza e i due volumi che contengono rispettivamente le conferenze ai preti della missione e le conferenze alle figlie della carità. Di prossima pubblicazione il testo italiano del vol. XIII dell'edizione francese del Coste, contenente Documenti.

Interessanti anche i volumetti della Collana Cháris-Oikós, agili strumenti di conoscenza di aspetti della spiritualità vincenziana.

3. La diffusione delle pubblicazioni del CLV avviene attraverso una rete di promozione e distribuzione libraria, ma si può accedere anche direttamente al sito web, sia per consultare e avere davanti l'intero panorama della produzione del Centro, sia anche per eventuali ordinazioni di testi. 